\title{
Chemical characterization and antioxidant activity of three medicinal Apiaceae species
}

\author{
Maulidiani ${ }^{a}$, Faridah Abas ${ }^{a, b}$, Alfi Khatib ${ }^{a, c}, K^{2}$ Khozirah Shaari ${ }^{\text {a,d }}$, Nordin H. Lajis ${ }^{a, e, *}$ \\ a Laboratory of Natural Products, Institute of Bioscience, Universiti Putra Malaysia, 43400 UPM, Serdang, Selangor, Malaysia \\ b Department of Food Science, Faculty of Food Science and Technology, Universiti Putra Malaysia, 43400 UPM, Serdang, Selangor, Malaysia \\ ${ }^{c}$ Department of Pharmaceutical Chemistry, Faculty of Pharmacy, International Islamic University Malaysia, Kuantan 25200, Pahang, Malaysia \\ d Department of Chemistry, Faculty of Science, Universiti Putra Malaysia, 43400 UPM, Serdang, Selangor, Malaysia \\ e Scientific Chairs Unit, Taibah University, P.O. Box 30001, Madinah al-Munawarah, Saudi Arabia
}

\section{A R T I C L E I N F O}

\section{Article history:}

Received 24 September 2013

Received in revised form 10 February 2014

Accepted 11 February 2014

Available online 15 March 2014

\section{Keywords:}

C. asiatica

H. bonariensis

H. sibthorpioides

HPLC-DAD-ESIMS

\begin{abstract}
A B S T R A C T
Centella asiatica ('Pegaga'= Malaysia) is well known plant that has been used as one of the ingredients in pharmaceutical and cosmetic industries. Using liquid chromatography with diode array and electrospray ionization/mass spectrometry (LC-DAD-ESI/MS) analysis, chemical profiling of three different Pegaga extracts (C. asiatica, $H$. bonariensis and $H$. sibthorpioides) revealed variations in their metabolite profile. Our findings showed that triterpenes of $C$. asiatica were characterized by the ursane-type triterpenes (madecassoside, asiaticoside, madecassic acid, and asiatic acid), while the two Hydrocotyle species consisted of oleanane-type triterpenes (barrigenol derivatives). Other variations are due to the difference in phenolic and flavonoid constituents. The three Pegaga extracts were also evaluated for their total phenolic content (TPC), 1,1-diphenyl-2-picrylhydrazyl (DPPH) free radicals, and xanthine oxidase inhibition (XOI) activities. The results showed that $C$. asiatica has the most potent antioxidant activity (TPC $=72.09 \mathrm{mg} / 100 \mathrm{~g}$ DW; $\mathrm{DPPH}=72.99 \mu \mathrm{g} / \mathrm{ml} ; \mathrm{XOI}=87.68 \mu \mathrm{g} / \mathrm{ml}$ ) as compared to H. bonariensis $(\mathrm{TPC}=28.55 \mathrm{mg} / 100 \mathrm{~g} \mathrm{DW} ; \mathrm{DPPH}=22.43 \mu \mathrm{g} / \mathrm{ml} ; \mathrm{XOI}=32.23 \mu \mathrm{g} / \mathrm{ml}$ ) and $H$. sibthorpioides $(\mathrm{TPC}=56.23 \mathrm{mg} / 100 \mathrm{~g} \mathrm{DW} ; \mathrm{DPPH}=37.86 \mu \mathrm{g} / \mathrm{ml} ; \mathrm{XOI}=14.73 . \mu \mathrm{g} / \mathrm{ml})$.
\end{abstract}

(c) 2014 Elsevier B.V. All rights reserved.

\section{Introduction}

Pegaga (Malaysian Pennywort) are perennial creeping plants of the genus Centella and Hydrocotyle of the Apiaceae family (De Padua and Bunyapraphatsara, 1999). In Malaysia, Pegaga, is valued medicinally and also consumed as a traditional vegetable or 'ulam'. Common varieties of Pegaga are Centella asiatica (asiatic pennywort), Hydrocotyle bonariensis (large leaf marsh pennywort), and Hydrocotyle sibthorpioides (lawn marsh pennywort). C. asiatica has been used since prehistoric time as a herbal remedy for a wide range of applications such as treatment of skin diseases and as a tonic for improving youth, longevity and memory (Burkill, 1966). In the pharmaceuticals industry, $C$. asiatica is a dermatologically effective phytotherapeutic agent that has been used in the form of complex

\footnotetext{
* Corresponding author at: Laboratory of Natural Products, Institute of Bioscience, Universiti Putra Malaysia, 43400 UPM, Serdang, Selangor, Malaysia. Tel.: +603 89471248; fax: +: 60389472218

E-mail addresses: khozirah@upm.edu.my (K. Shaari),nordinlajis@gmail.com (N.H. Lajis).
}

homeopathic preparations, such as an ointment for external application (Brinkhaus et al., 2000). C. asiatica has been also used in the cosmetics industry, e.g. as anti-ageing specifically for ameliorating lines and wrinkles, and prophylactically treating loss of elasticity (Oblong and Bissett, 1997), and improving keratinocytes differentiation and for enhancing the epidermal functionality (Sene et al., 2007).

The phytochemistry (Rastogi et al., 1960; Asakawa et al., 1982; Matsuda et al., 2001) and pharmacology (Brinkhaus et al., 2000) of $C$. asiatica has been extensively studied. The main compounds that have been reported to exhibit various physiological effects include its characteristic triterpenoids asiaticoside, madecassoside, asiatic acid and madecassic acid (Gunther and Wagner, 1996). Other classes of compounds such as sterols, polyacetylenes (Govindan et al., 2007), flavonoids and chlorogenic acids (Satake et al., 2007) were also reported to be present in C. asiatica.

Despite its popular use, a great deal of uncertainly still exist with regards to the differences between the Pegaga varieties, presenting problems in quality control and standardization of downstream products derived from the plants. Earlier, we were able to differentiate between the three Pegaga varieties $C$. asiatica, $H$. bonariensis, 
and $H$. sibthorpioides by using ${ }^{1} \mathrm{H}$ NMR metabolomics approach (Maulidiani et al., 2012). The study revealed that asiaticoside and madecassoside along with chlorogenic acids were the metabolites contributing to the separation of $C$. asiatica, $H$. bonariensis and $H$. sibthorpiodes extracts. The two triterpene glycosides were only detected in $C$. asiatica extracts but not in $H$. bonariensis, and $H$. sibthorpioides. These compounds are well known to exhibit important biological activities including antioxidant, anticancer and anti-inflammation (Al-Saeedi et al., 2011; Wan et al., 2012).

${ }^{1} \mathrm{H}$ NMR spectroscopy has been used widely as an analytical tool because of its robustness, speed and high-throughput as well as the relatively simple method of sample preparation (Lindon and Nicholson, 1997; Kim et al., 2010). However, MS-based techniques are also widely used as it offers greater speed, sensitivity and selective qualitative and quantitative analyses. Furthermore, efficient identification of each individual constituent is enabled by application of tandem mass (MS/MS) (Schutz et al., 2004; Seeram et al., 2006).

Numerous reports have been published on the quantification of major triterpenoids from $C$. asiatica (asiaticoside, madecassoside, asiatic acid, madecassic acid and their isomers) using HPLC (Gunther and Wagner, 1996; Schaneberg et al., 2003; Rafamantanana et al., 2009). However, there has been only a few reports on the identification of $C$. asiatica constituents using LCMS (Shen et al., 2009). Recently, Long et al. (2012) reported the online identification of two Centella species using LCMS. In this paper, we report the on-line identification of three Pegaga varieties $(C$. asiatica, $H$. bonariensis, and $H$. sibthorpioides) using LCMS. The diagnostic fragmentation patterns of chlorogenic acids, flavonoids, and triterpenes in the ESI-MS/MS are discussed on the basis of MS/MS data, in comparison with literature and authentic standards. We further compared the antioxidant and xanthine oxidase inhibitory activities of the three Pegaga varieties.

\section{Materials and methods}

\subsection{Chemicals}

Ethanol 70\% (v/v) used as a solvent for extraction was purchased from ChemPur (Karlsruhe, Germany). HPLC grade acetonitrile (Merck, Darmstadt, Germany) and water purified by a MilliQ system (Millipore, Bedford, MA, USA) were used as mobile phase solvent system in the LCMS analysis. Acetic acid (HOAc) was used as buffer (Fisher, Loughborough, UK). Asiaticoside, madecassoside, asiatic acid, and madecassic acid were purchased from Sigma Chemical Co. (USA), while quercetin and kaempferol were isolated from the dichloromethane fraction of $C$. asiatica and unambiguously identified structurally by ${ }^{1} \mathrm{H}$ NMR analysis and comparison with literature data (Satake et al., 2007). Gallic acid, Folin-Ciocalteu reagent, and sodium carbonate 1,1-diphenyl-2picrylhydrazyl (DPPH), xanthine oxidase (EC 1.17.3.2), xanthine, potassium phosphate monobasic anhydrous, and allopurinol were purchased from Sigma Chemical Co. (St. Louis, MO, USA). Standard compounds 3-O-caffeoylquinic acid (chlorogenic acid) and quercetin-3-O-rhamnoside were also purchased from Sigma Chemical Co. (St. Louis, MO, USA).

\subsection{Plant materials}

Leaves of three varieties of Pegaga (C. asiatica, H. bonariensis, and $H$. sibthorpioides) were collected from the plant nursery of the Institute of Bioscience, Universiti Putra Malaysia in October 2008. The three varieties were identified by a Resident Botanist and voucher specimens were deposited at the herbarium of the Laboratory of Natural Products, University of Putra Malaysia.

\subsection{Extraction}

One gram of the air-dried powdered plant material was soaked in $50 \mathrm{ml}$ of $70 \%$ aqueous ethanol (v/v). The suspension was ultrasonicated at room temperature for $60 \mathrm{~min}$, filtered, concentrated in vacuo and lyophilized. Extracts were kept at $4{ }^{\circ} \mathrm{C}$ prior to analysis.

\subsection{HPLC-DAD-ESIMS}

Mass spectra were acquired using a Thermo-Finnigan model $\mathrm{LCQ}^{\mathrm{DECA}}$ (San Jose, $\mathrm{CA}$ ) ion-trap mass spectrometer equipped with an ESI source interface. Ultrahigh purity helium (He) and high purity nitrogen $\left(\mathrm{N}_{2}\right)$ were used as collision and nebulizing gases, respectively. The mass spectra were acquired in both positive and negative ion modes in separate analyses with the following conditions: ESI cone voltage $\pm 4 \mathrm{kV}$; heated capillary temperature $235^{\circ} \mathrm{C}$, sheath and auxiliary nitrogen gas flows 40 and 18 units. The instrument was coupled to a Surveyor HPLC binary pump, Surveyor diode array detector (DAD) (190-600 nm range; $1 \mathrm{~nm}$ bandwidth) and Surveyor autosampler. Chromatographic separation was carried out on an XTerra column $(3.5 \mu \mathrm{m}, 150 \times 3 \mathrm{~mm}$ i.d., Waters Corporation, USA). Acquisition time for the analysis was $80 \mathrm{~min}$ using gradient elution with acetonitrile $(A)$ and water containing 0.5\% HOAc (B): 0-5 min, 10\% A; 5-15 min, 20-25\% A; 15-20 min, 25-30\% A; 20-35 min, 30\% A; 35-40 min, 30-40\% A; 40-45 min, 40-55\% A; 45-50 min, 55-70\% A; 50-55 min, 70-80\%A; 55-60 min, $80-90 \%$ A; 60-65 min, 90-100\% A; 65-80 min, 100\%A at a flow-rate of $0.5 \mathrm{ml} / \mathrm{min}$. The concentration of sample extract for each analysis is $5 \mathrm{mg} / \mathrm{ml}$ (injection volume: $10 \mu \mathrm{l}$ ). The total ion chromatograms (TIC) were recorded for $\mathrm{m} / \mathrm{z} 50$ to $\mathrm{m} / \mathrm{z}$ 2000. A data-dependent program was used for further MS/MS analysis. Quantitative determination of antioxidant compounds was carried out via HPLC-DAD analysis. Quercetin and quercetin-3-O-rhamnoside were selected as the external standards for quantification of flavonoids, whereas 3-O-caffeoylquinic acid (chlorogenic acid) for caffeoylquinic acid derivatives. Relative quantification was determined using calibration curves constructed from the standards, prepared in five different concentration levels ranging from 0.1 to $0.006 \mathrm{mg} / \mathrm{ml}$. The calibration curve criteria must be accepted if the $R^{2} \geq 0.99$. The relative amount of the compound was expressed as $\mathrm{mg} / 100 \mathrm{~g}$ of extract.

\subsection{Total phenolic content (TPC)}

The total phenolic content (TPC) was determined using FolinCiocalteu method (Singleton et al., 1999). In this method, $0.1 \mathrm{ml}$ extract $(1 \mathrm{mg} / \mathrm{ml})$ was reacted with $0.1 \mathrm{ml}$ Folin-Ciocalteu reagent and then neutralized with $10 \mathrm{ml}$ of sodium carbonate $(7 \%, \mathrm{v} / \mathrm{v}$; in distilled water). After 60 min of incubation, the absorbance of the solution was measured at $725 \mathrm{~nm}$. Gallic acid was used as a standard and the TPC was expressed as mg of gallic acid equivalents/100 $\mathrm{g}$ sample DW.

\subsection{1,1-Diphenyl-2-picrylhydrazyl radical (DPPH) activity}

Radical scavenging activity using >1,1-diphenyl-2picrylhydrazyl radical (DPPH) was carried out according to protocols described by Mohamad et al. (2004). $100 \mu$ l of substock solution (in $\mathrm{MeOH}$ ) of the extracts at concentrations 250, 125, and $62.5 \mu \mathrm{g} / \mathrm{ml}$ were added into 96 -well microplates.5 $\mu \mathrm{l}$ of DPPH reagent ( $5 \mathrm{mg}$ of DPPH in $2 \mathrm{ml}$ of $\mathrm{MeOH}$ ) was then added into each well and the solution was kept in the dark for $30 \mathrm{~min}$. The absorbance of the solution was then measured at $517 \mathrm{~nm}$. 
Percentage inhibition of DPPH activity was calculated based on the following formula:

$$
\frac{\left(\mathrm{OD}_{\text {control }}-\mathrm{OD}_{\text {samplex }}\right) \times 100 \%}{\mathrm{OD}_{\text {control }}}
$$

\subsection{Xanthine oxidase inhibitory (XOI) activity}

The assay was conducted based on previously reported procedure (Sweeney et al., 2001) with slight modifications. The samples were tested at the concentrations of $200,100,50,25$, and $12.5 \mu \mathrm{g} / \mathrm{ml}$. Percentage inhibition of XOI activity was calculated according to the formula:Percentage inhibition $=\left(1-\left(\mathrm{OD}_{\text {sample }}-\right.\right.$ $\left.\left.\mathrm{OD}_{\text {control }}\right)\right) \times 100 \%$

\subsection{Statistical analysis}

All experiments were repeated thrice and the results reported as mean \pm SEM values. Data were analyzed using ANOVA with Tukey-HSD pairwise comparison using Statistical Package for Social Science $\left(\right.$ SPSS $^{\mathrm{TM}}$ ) software for Windows, Version 16.0 (SPSS Inc., Chicago, IL). A probability level of $5 \%(p \leq 0.05)$ was considered as significant.

\section{Results and discussion}

\subsection{Liquid chromatography mass spectrometry analysis}

The HPLC-DAD chromatograms of the three Pegaga variaties ( $C$. asiatica, $H$. bonariensis, and $H$. sibthorpioides) recorded in the UV range of $190-600 \mathrm{~nm}$ showed distinctly different metabolite profiles (Fig. 1). The chromatograms of each extract, recorded over $80 \mathrm{~min}$, indicated that the major components were eluted between 5 and $50 \mathrm{~min}$. The TIC profiles showed that most of the prominent peaks detected were attributable to the presence of chlorogenic acids, flavonoids, and triterpene glycosides (Fig. 2). As expected, the triterpenoids, generally having poor chromophores, were poorly detected within the wavelength range of the PDA detector (Fig. 1).

Chlorogenic acids were detected in both $C$. asiatica and $H$. sibthorpioides extracts, whereas flavonoids were present in all of the extracts. Triterpene glycosides were detected in all of the extracts but the aglycones were different for each of the test extract. Table 1 summarizes the retention times (RT), UV, MS and MS/MS data of the detected metabolites.

\subsection{Identification of phenolic acids}

Chlorogenic acids are a family of esters of hydroxycinnamic acids (caffeic acid, ferulic acid and $p$-coumaric acid) with quinic acids. Based on the negative ion MS/MS data (Table 1 ), caffeic acid $(m / z 179)$ and ferulic acid $(m / z 193)$ appear to be the common type of hydroxycinnamic acids for the chlorogenic acids of C. asiatica and $H$. sibthorpioides, respectively. Meanwhile, the fragment ion at $m / z 191$ was characteristic of quinic acid. The ions at $\mathrm{m} / \mathrm{z} 191$ and 179 were considered to be generated from the cleavage of the ester bond linking the two acids. 3-O-Caffeoylquinic acid (chlorogenic acid) was observed early at the retention time of 4.91 min (peak 1) in the TIC profile of both $C$. asiatica and $H$. sibthorpioides. Meanwhile, dicaffeoylquinic acids appear to be major components of the $C$. asiatica extract. This was evident from the negative ion MS/MS data for peaks 11-13, 15, and 17 of $C$. asiatica extract (Fig. 2A) which exhibited characteristic ions at $\mathrm{m} / \mathrm{z}$ 191, 353, and 515 (Table 1) attributable to quinic, chlorogenic, and dicaffeoyl quinic acid (cynarin) moieties, respectively. Peaks 11, 12 and 15 exhibited similar molecular ion peaks at $\mathrm{m} / \mathrm{z} 515$ indicating that they are possibly isomers, tentatively identified as 1,5-di-O-caffeoylquinic acid, 3,5-di-O-caffeoylquinic acid and 4,5-di-O-caffeoylquinic acid based on comparison with previous reports (Schutz et al., 2004). Peaks 13 and 17 also showed the same molecular ion peak at $m / z 601$ and based on its MS/MS fragmentation with $\mathrm{m} / \mathrm{z}$ 515, 395 and 353, these were tentatively assigned as isomers of 3,5-di-O-caffeoyl-4-malonylquinic acid (Lin and Harnly, 2010).

Possible isomers of dehydrotriferulic acids were identified at the retention times of $35.56 \mathrm{~min}$ (peak 29) and $37.71 \mathrm{~min}$ (peak 33), for $H$. sibthorpioides. The assignments were based on the deprotonated molecular ion peak at $m / z 577$, suggesting a molecular weight of 578 and the fragment ions at $m / z 383$ and 193, corresponding to the loss of one and two ferrulic acid moieties, respectively. The UV maxima at $320 \mathrm{~nm}$ shown by both compounds were also characteristic of ferulic acid derivatives and comparison with literature data further supported the assignment (Funk et al., 2005).

\subsection{Identification of flavonoid derivatives}

Fig. 1 reveals that quercetin and kaempferol were the major flavonols found in the Pegaga extracts. Quercetin was characterized by characteristic absorption maxima at 255 and $350 \mathrm{~nm}$, whereas kaempferol was characterized based on its absorption maxima at 260 and $340 \mathrm{~nm}$. In C. asiatica extract, three flavonoids could be identified, which were quercetin 3-O- $\beta$-D-glucuronide, quercetin and kaempferol at RTs=12.01 $\mathrm{min}$ (peak 8), $27.83 \mathrm{~min}$ (peak 24), and $35.84 \mathrm{~min}$ (peak 30), respectively. Peak $\mathbf{8}$ exhibited a deprotonated molecular ion at $m / z 477$ with fragment ions at $m / z 301$ for the characteristic loss of the glucuronyl moiety, followed by the typical fragment ions $(\mathrm{m} / \mathrm{z} 179$ and 151$)$ of free quercetin.

Hydrocotyle species contained flavonoid glycosides as likely chemical markers. Rutin (peak 3) was identified by the deprotonated molecular ion at $m / z 609$ and the fragment ions at $\mathrm{m} / z 463$ (for the loss of rhamnose, $[\mathrm{M}-146]^{-}$) and 301 (loss of rhamnose and glucose $\left.[\mathrm{M}-146-162]^{-}\right)$. Meanwhile peak $4(\mathrm{RT}=10.25 \mathrm{~min})$ with a deprotonated molecular ion peak at $m / z 595$ was tentatively assigned as quercetin- $O$-pentosyl-hexoside (Singh et al., 2011 ) based on the fragment ions at $m / z 463$ (loss of pentose moiety [M-132]) and 301 (loss of pentose and glucose [M-132-162 $]^{-}$). Peak 6 was assigned as kaempferol-3-O-rutinoside ( $R T=11.29 \mathrm{~min}$ ) based on the molecular ion at $m / z 593$ and fragment ions at $m / z$ 447 (loss of rhamnose [M-H-146] $]^{-}$) and 284 (loss of rhamnose and glucose [M-146-162 $]^{-}$. Peak 7 (RT=11.73 min) was identified as quercetin-3-O-glucoside based on the deprotonated molecular ion at $m / z 463$, and fragment ions at $m / z 445$ (loss of $\mathrm{H}_{2} \mathrm{O}[\mathrm{M}-18]^{-}$) and $>301$ (loss of glucoside). These flavonoid glycosides were also major components of $H$. sibthorpioides. Peak $14(\mathrm{RT}=15.18 \mathrm{~min})$ present in $H$. sibthorpioides extract exhibited a deprotonated molecular ion at $m / z$ 447. Further MS/MS analysis gave a fragment ion at $m / z 301$ for [M-H-146] $]^{-}$arising from a loss of rhamnose yielding the aglycone, quercetin, based on the fragments at $\mathrm{m} / \mathrm{z} 271,255,179$ and 151. Based on this the compound was assigned as quercetin-3-Orhamnoside.

A larger number of flavonol glycosides were detected in $H$. sibthorpioides extract. A deprotonated molecular ion at $\mathrm{m} / \mathrm{z} 741$ for peak 2 (RT $=9.08 \mathrm{~min}$ ) and its fragment ions at $m / z 609$, 447 , and 301 , which were attributable to loss of pentose [M-H$132]^{-}$, glucose $[\mathrm{M}-\mathrm{H}-162]^{-}$and rhamnose $[\mathrm{M}-\mathrm{H}-146]^{-}$from the parent molecular ion, respectively, indicating it to be quercetinrutinoside with an additional pentose moiety (Ferreres et al., 2010). An isomer of quercetin-rutinoside was also detected as peak $\mathbf{5}$ ( $\mathrm{RT}=10.85 \mathrm{~min}$ ) based on the deprotonated ion peak at $\mathrm{m} / z 609$. Peak 10 ( $R T=13.92 \mathrm{~min}$ ) with a deprotonated molecular ion peak at $m / z$ at 447 , identified as kaempferol-3-O-glucoside. Peak 18 ( RT = $18.63 \mathrm{~min}$ ) was identified as quercetin-3-diglucoside based on 
RT: $0.00-50.00$ SM: $15 \mathrm{G}$

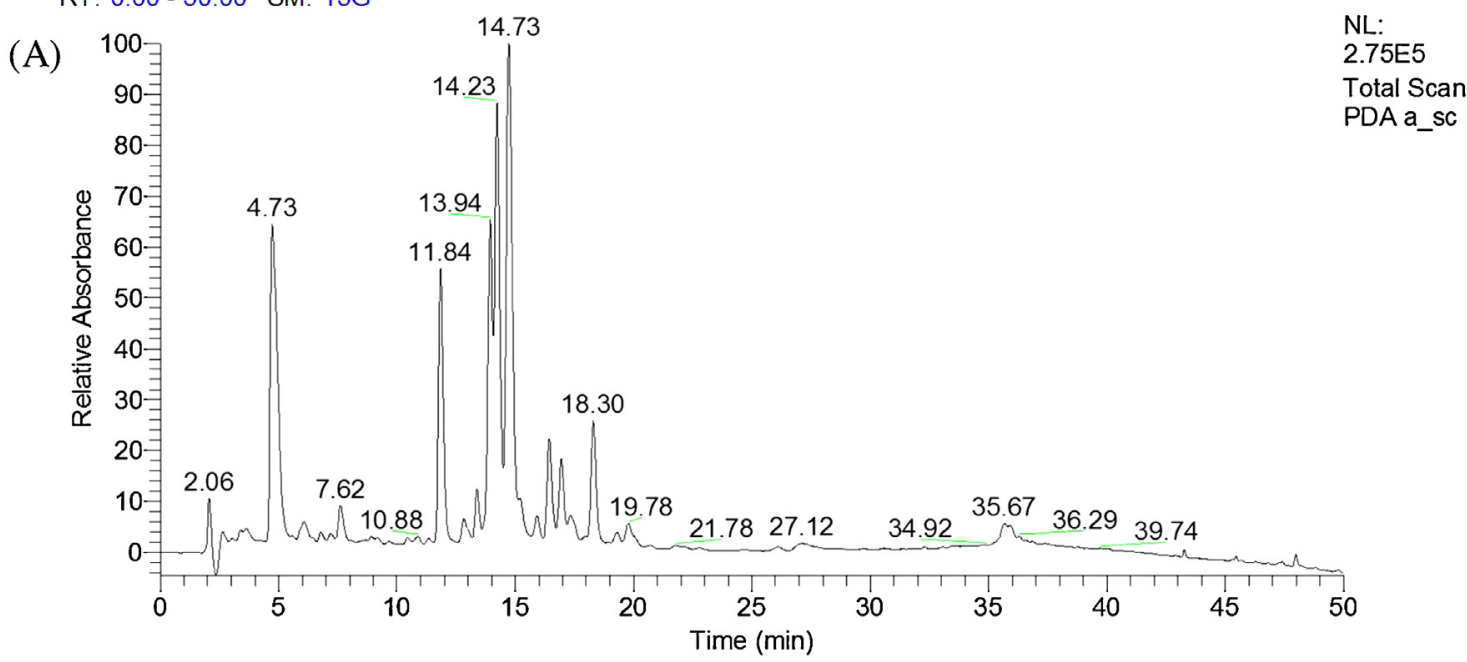

RT: $0.00-50.00$

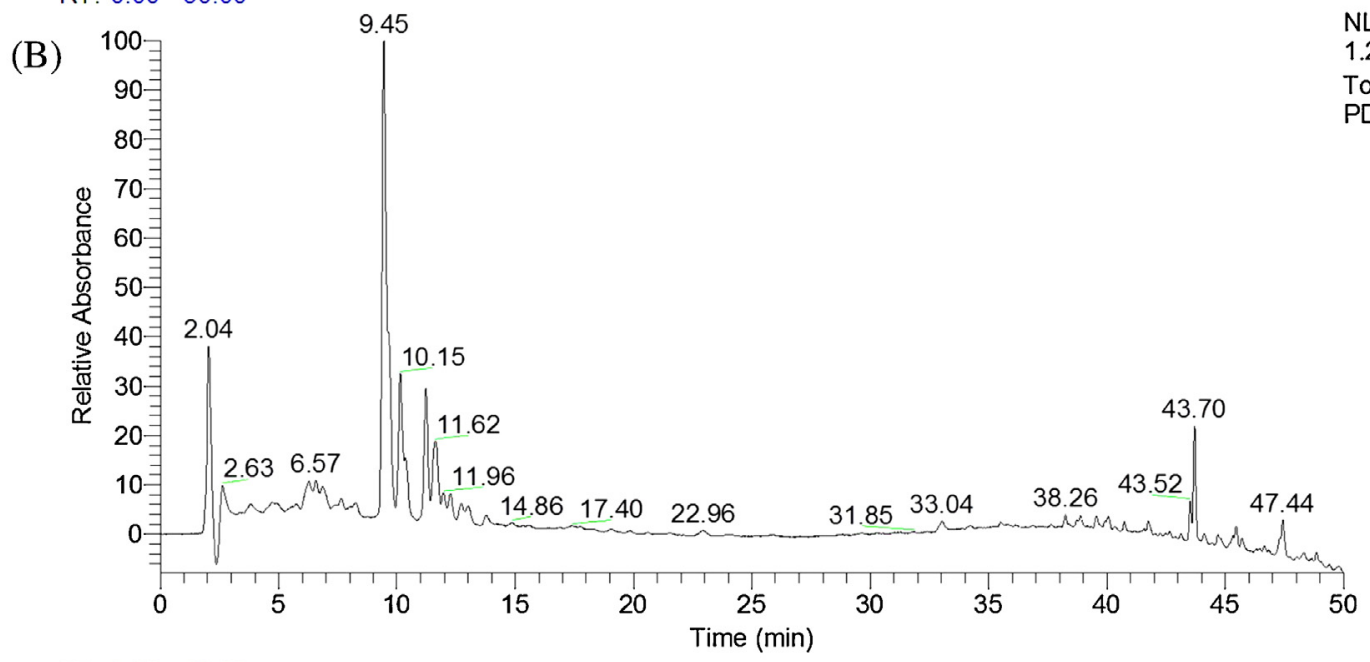

RT: $0.00-50.00$

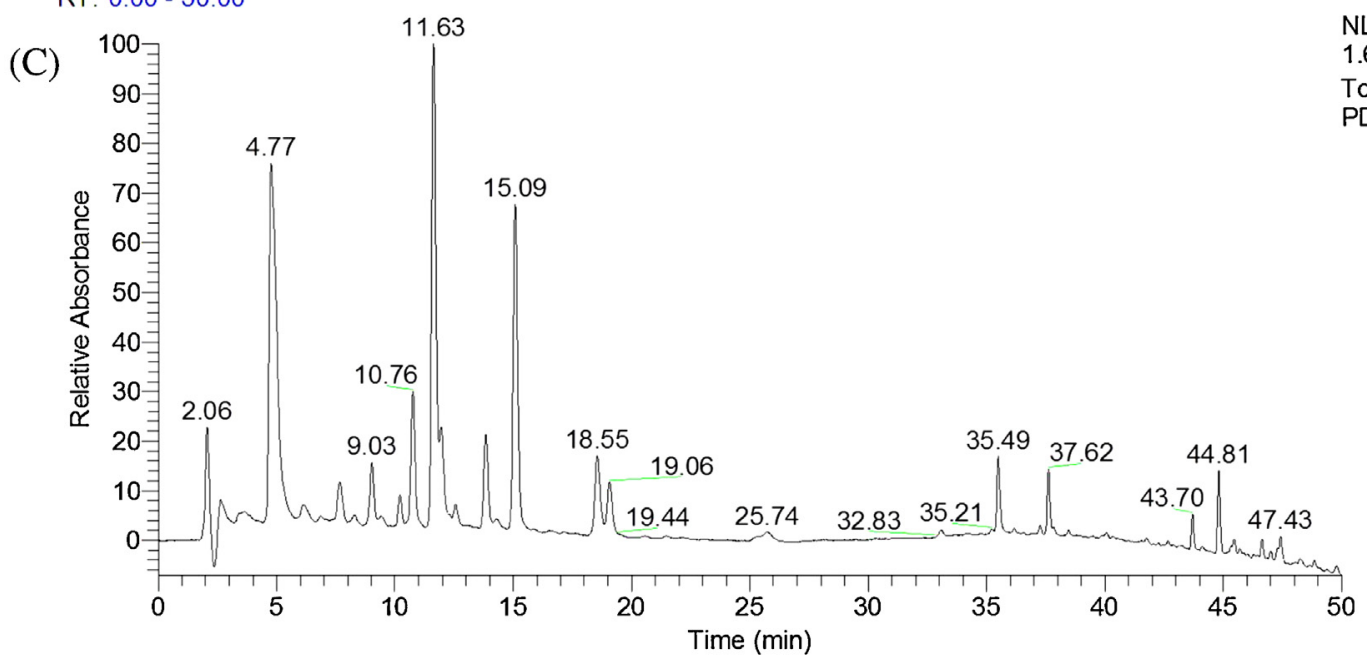

Fig. 1. HPLC-DAD chromatograms of (A) C. asiatica, (B) H. bonariensis, and (C) H. sibthorpioides.

NL:

27 E5

Total Scan

PDAb_sc

NL:

1.63E5

Total Scan

PDA c_sc 
RT: $0.00-50.00$ SM: $15 \mathrm{G}$

(A)

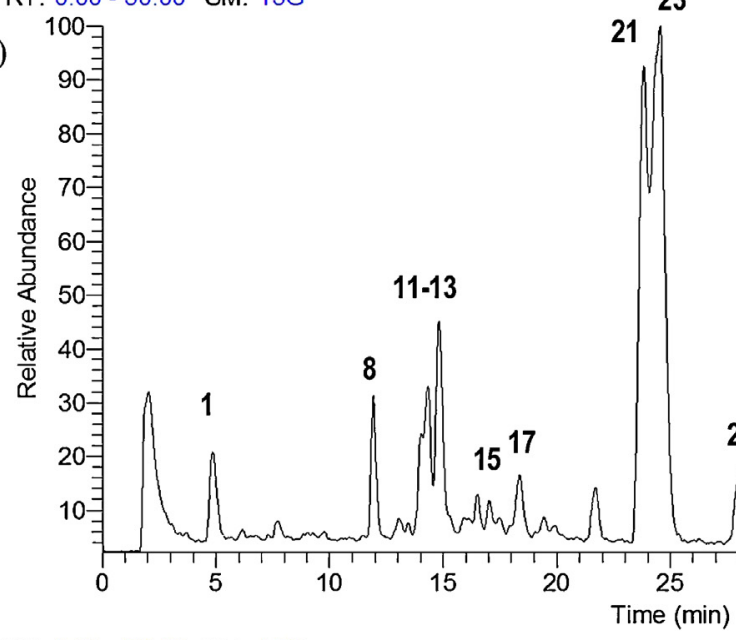

23

NL:

5.92E9

TIC MS

a_sc

RT: $0.00-50.00$ SM: $15 G$

(B)

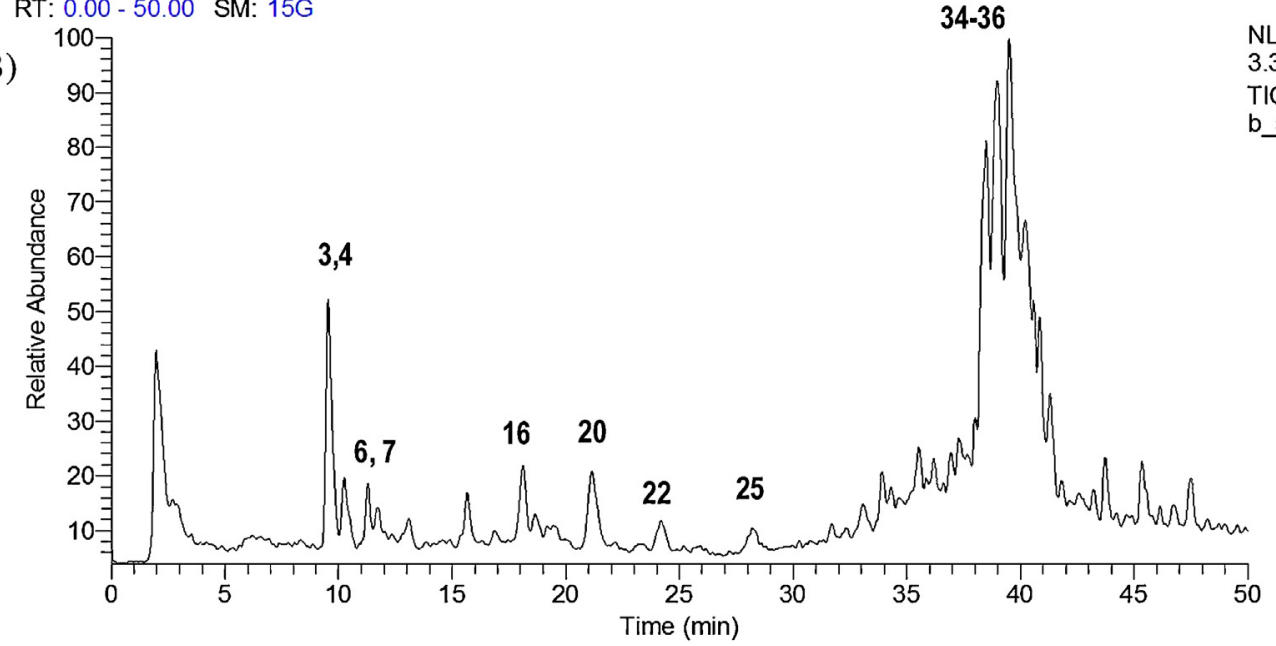

NL:

TIC MS

b_sc

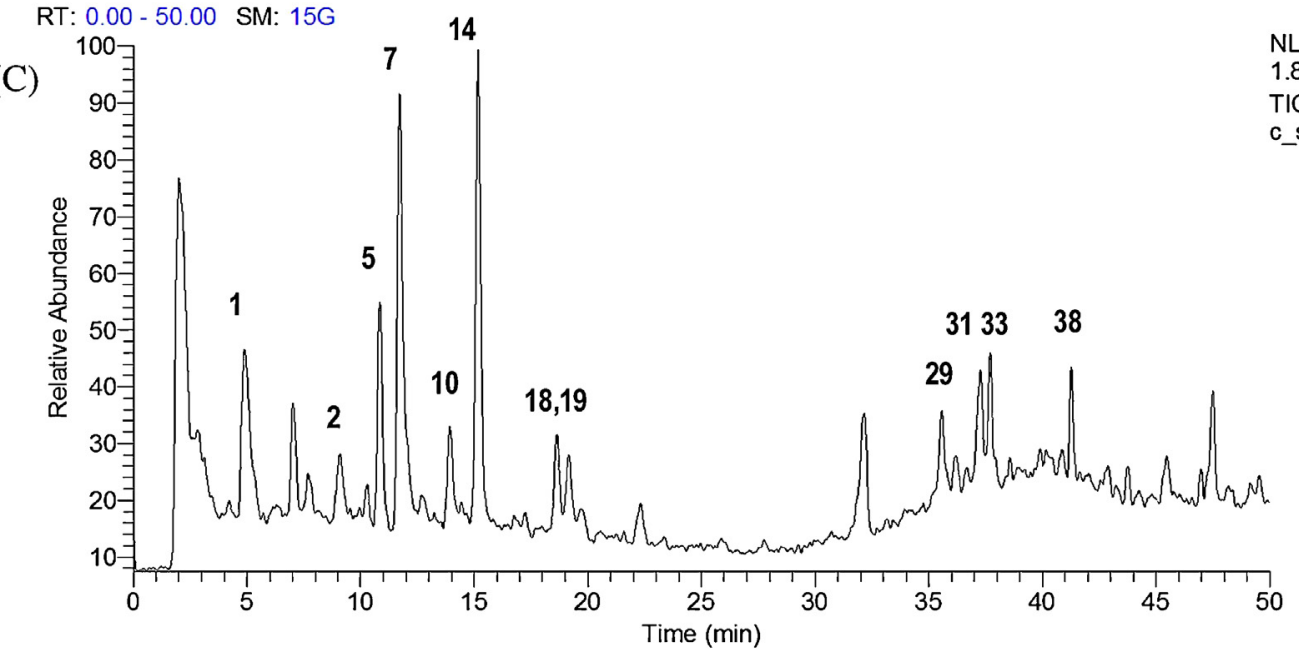

Fig. 2. Total ion chromatograms of (A) C. asiatica, (B) H. bonariensis, and (C) H. sibthorpioides. For peak assignments, see Table 1. 
Table 1

Identification of phenolic, flavonol, and triterpenoid constituents in Pegaga extracts based on their spectral characteristics obtained from negative ion LC-DAD-ESIMS data.

\begin{tabular}{|c|c|c|c|c|c|c|c|c|c|}
\hline \multirow[t]{2}{*}{ Peak no. } & \multirow[t]{2}{*}{$\begin{array}{l}\text { Retention } \\
\text { time (min) }\end{array}$} & \multirow[t]{2}{*}{$\mathrm{UV}(\mathrm{nm})$} & \multirow[t]{2}{*}{$\begin{array}{l}\text { Parent ion (negative } \\
\text { ion) }\end{array}$} & \multirow[t]{2}{*}{$\begin{array}{l}\mathrm{MS}^{2} \text { fragments (base } \\
\text { ion in bold) }\end{array}$} & \multirow[t]{2}{*}{ Tentative identity of compound } & \multirow[t]{2}{*}{$\begin{array}{l}\text { Pegaga } \\
\text { extract }^{\mathrm{a}}\end{array}$} & \multicolumn{3}{|c|}{$\begin{array}{l}\text { Relative amounts of antioxidant } \\
\text { compounds }^{\mathrm{b}}(\mathrm{mg} / 100 \mathrm{~g} \text { extract })\end{array}$} \\
\hline & & & & & & & CA & HB & $\mathrm{HS}$ \\
\hline \multicolumn{10}{|c|}{ Phenolics } \\
\hline 1 & 4.91 & 235,320 & 353 & 191, 179, 135 & $\begin{array}{l}\text { 3-O-Caffeoylquinic acid (Chlorogenic } \\
\text { acid) }\end{array}$ & $\mathrm{CA}, \mathrm{HS}$ & $0.033 \pm 3.284^{c}$ & - & $0.018 \pm 3.784^{c}$ \\
\hline 11 & 14.19 & 245,325 & 515 & $\mathbf{3 5 3}, 335,191,179$ & 1,5-Di-O-caffeoylquinic acid & CA & $0.030 \pm 4.323^{c}$ & - & - \\
\hline 12 & 14.44 & 245,325 & 515 & $\mathbf{3 5 3}, 335,191,179$ & 3,5-Di-O-caffeoylquinic acid & CA & $0.020 \pm 4.344^{c}$ & - & - \\
\hline 13 & 14.99 & 245,325 & 601 & $557, \mathbf{5 1 5}, 395,353,233$ & $\begin{array}{l}\text { 3,5-Di-O-caffeoyl-4- } \\
\text { malonylquinic acid } \\
\text { (Irbic acid) }\end{array}$ & CA & $0.023 \pm 5.838^{c}$ & - & - \\
\hline 15 & 16.70 & 245,325 & 515 & $\mathbf{3 5 3}, 191,179,173$ & 4,5-Di-O-caffeoylquinic acid & CA & $0.007 \pm 5.141^{c}$ & - & - \\
\hline 17 & 18.51 & 245,325 & 601 & $557,515, \mathbf{3 9 5}, 245$ & $\begin{array}{l}\text { 3,5-Di-O-caffeoyl-4-malonylquinic acid } \\
\text { (Irbic acid) isomer }\end{array}$ & CA & $0.006 \pm 6.535^{c}$ & - & - \\
\hline 29 & 35.56 & 245,320 & 577 & $549,505, \mathbf{3 8 3}, 355,313,193,163$ & Dehydrotriferulic acid & HS & - & - & - \\
\hline 33 & 37.71 & 250,320 & 577 & $\mathbf{5 3 3}, 505,383,355,341,298,193,163$ & Dehydrotriferulic acid & HS & - & - & - \\
\hline \multicolumn{10}{|c|}{ Flavonols } \\
\hline 2 & 9.08 & 250,345 & 741 & $651,609,447, \mathbf{3 0 1}, 271,255$ & $\begin{array}{l}\text { Quercetin-rutinoside analogue with an } \\
\text { additional pentose moiety }\end{array}$ & HS & - & - & $0.048 \pm 4.534^{\mathrm{d}}$ \\
\hline 3 & 9.54 & 255.350 & 609 & $463, \mathbf{3 0 1}, 271,255,179$ & Quercetin-rutinoside & $\mathrm{HB}$ & - & $0.423 \pm 2.855^{\mathrm{d}}$ & - \\
\hline 4 & 10.25 & 255,350 & 595 & $463, \mathbf{3 0 1}, 271,255,179$ & Quercetin-O-pentosyl-hexoside & HB & - & $0.105 \pm 3.545^{\mathrm{d}}$ & - \\
\hline 5 & 10.85 & 255,350 & 609 & $465, \mathbf{3 0 1}, 271,>255,179$ & Isomer of quercetin-rutinoside & HS & - & - & $0.098 \pm 3.831^{\mathrm{d}}$ \\
\hline 6 & 11.29 & 260,340 & 593 & $447, \mathbf{2 8 4}, 255,227,179 \mathrm{MS}^{3} \mathbf{2 5 5}, 163,151$ & $\begin{array}{l}\text { Kaempferol 3-O-glucoside } \\
\text { 7-O-rhamnoside }\end{array}$ & $\mathrm{HB}$ & - & $0.146 \pm 2.934^{\mathrm{d}}$ & - \\
\hline 7 & 11.73 & 255,350 & 463 & $445, \mathbf{3 0 1}, 271,255,179$ & Quercetin-3-O-glucoside & $\mathrm{HB}, \mathrm{HS}$ & - & $0.085 \pm 2.572^{\mathrm{d}}$ & $0.296 \pm 1.092^{\mathrm{d}}$ \\
\hline 8 & 12.01 & 255,350 & 477 & $\mathbf{3 0 1}, 179,151$ & Quercetin 3-O-b-D-glucuronide & $\mathrm{CA}$ & $0.677 \pm 2.804^{d}$ & - & - \\
\hline 10 & 13.92 & 260,340 & 447 & $\mathbf{2 8 4}, 255,227,179,151$ & Kaempferol-3-O-glucoside & HS & - & - & $0.062 \pm 3.290^{\mathrm{d}}$ \\
\hline 14 & 15.18 & 255,345 & 447 & $\mathbf{3 0 1}, 271,255,179,151$ & Quercetin-3-O-rhamnoside & HS & - & - & $0.228 \pm 2.273^{\mathrm{d}}$ \\
\hline 18 & 18.63 & 250,330 & 625 & $463,301,179$ & Quercetin-3-diglucoside & HS & - & - & $0.040 \pm 7.814^{\mathrm{d}}$ \\
\hline 19 & 19.17 & 260,335 & 431 & $\mathbf{2 8 5}, 255,227,191,179$ & Kaempferol 3-O-rhamnoside & HS & - & - & $0.035 \pm 8.950^{\mathrm{d}}$ \\
\hline 24 & 27.56 & 255,345 & 301 & $301,273,257,239, \mathbf{1 7 9}, 151,125$ & Quercetin & CA & $0.218 \pm 1.359^{d}$ & - & - \\
\hline 30 & 35.84 & 260,365 & 285 & $\mathbf{2 8 5}, 256,229,169$ & Kaempferol & CA & $0.193 \pm 4.313^{\mathrm{d}}$ & - & - \\
\hline \multicolumn{10}{|c|}{ Triterpenes } \\
\hline 16 & 18.12 & - & $\begin{array}{l}1021.2[\mathrm{M}+\mathrm{HOAc}- \\
\mathrm{H}] \rightarrow 961.5\end{array}$ & $\begin{array}{l}\text { 799.5, 667.5, 505, 457, } \\
421\end{array}$ & Ranuncoside II & $\mathrm{HB}$ & - & - & - \\
\hline 20 & 21.15 & - & $\begin{array}{l}859 \\
{[\mathrm{M}+\mathrm{HOAC}-} \\
\mathrm{H}] \rightarrow 799.5\end{array}$ & $\begin{array}{l}\mathrm{MS}^{2} \mathbf{7 9 9 . 5}, 505 \\
\mathrm{MS}^{3} \mathbf{6 6 7}, 505,437,421\end{array}$ & Ranuncoside I & $\mathrm{HB}$ & - & - & - \\
\hline 21 & 23.6 & - & $\begin{array}{l}1033 \\
{[\mathrm{M}+\mathrm{HOAc}-} \\
\mathrm{H}] \rightarrow 973\end{array}$ & $\begin{array}{l}\text { Full ms } \\
\text { 1033, 973, 929, 769, } \\
736,624,504,469,392, \\
347,291,256,179,119\end{array}$ & Madecassoside & CA & - & - & - \\
\hline 22 & 24.19 & & $\begin{array}{l}946 \\
{[\mathrm{M}+\mathrm{HOAC}-} \\
\mathrm{H}] \rightarrow 886\end{array}$ & $\begin{array}{l}\mathrm{MS}^{2} 813,751,685 \\
>663,619,601,533 \\
505,457 \\
\mathrm{MS}^{3} 619,601,551,505\end{array}$ & Barrigenol derivatives & $\mathrm{HB}$ & - & - & - \\
\hline 23 & 24.2 & - & $\begin{array}{l}1033 \\
{[\mathrm{M}+\mathrm{HOAC}-} \\
\mathrm{H}] \rightarrow 973\end{array}$ & $\begin{array}{l}\text { Full ms } \\
\text { 1033, } 973,965,782, \\
737,586,557,505,469, \\
375,287,189,177,119\end{array}$ & Asiaticoside B & CA & - & - & - \\
\hline 25 & 28.24 & - & $\begin{array}{l}813 \\
{[\mathrm{M}+\mathrm{HOAC}-} \\
\mathrm{H}] \rightarrow 753\end{array}$ & $\begin{array}{l}\mathrm{MS}^{2} 763,681,663,645, \\
601,573, \mathbf{5 3 3}, 505,455, \\
421,389 \\
\mathrm{MS}^{3}>501, \mathbf{4 6 7}, 456, \\
422\end{array}$ & Barrigenol derivatives & $\mathrm{HB}$ & - & - & - \\
\hline
\end{tabular}




\begin{tabular}{|c|c|c|c|c|c|c|c|c|c|}
\hline \multirow[t]{2}{*}{ Peak no. } & \multirow[t]{2}{*}{$\begin{array}{l}\text { Retention } \\
\text { time (min) }\end{array}$} & \multirow[t]{2}{*}{$\mathrm{UV}(\mathrm{nm})$} & \multirow[t]{2}{*}{$\begin{array}{l}\text { Parent ion (negative } \\
\text { ion) }\end{array}$} & \multirow[t]{2}{*}{$\begin{array}{l}\mathrm{MS}^{2} \text { fragments (base } \\
\text { ion in bold) }\end{array}$} & \multirow[t]{2}{*}{ Tentative identity of compound } & \multirow[t]{2}{*}{$\begin{array}{l}\text { Pegaga } \\
\text { extract }^{a}\end{array}$} & \multicolumn{3}{|c|}{$\begin{array}{l}\text { Relative amounts of antioxidant } \\
\text { compounds }^{\mathrm{b}}(\mathrm{mg} / 100 \mathrm{~g} \text { extract })\end{array}$} \\
\hline & & & & & & & CA & $\mathrm{HB}$ & HS \\
\hline 26 & 31.02 & - & $\begin{array}{l}1017 \\
{[\mathrm{M}+\text { HOAC- }} \\
\mathrm{H}] \rightarrow 957\end{array}$ & $\begin{array}{l}\mathbf{9 9 9}, 903,791,678,565 \\
452,338\end{array}$ & Centellasaponin A & CA & - & - & - \\
\hline 28 & 32.26 & - & $\begin{array}{l}1017 \\
{[\mathrm{M}+\mathrm{HOAC}-} \\
\mathrm{H}] \rightarrow 957.5\end{array}$ & $\mathbf{9 9 9}, 791,565$ & Asiaticoside & CA & - & - & - \\
\hline 31 & 37.27 & - & 1118 & $\begin{array}{l}\mathbf{9 5 6}, 893,793,775,757 \\
731,685,660,569,551, \\
456\end{array}$ & Hydrocotyloside III & HS & - & - & - \\
\hline 32 & 37.63 & - & $\begin{array}{l}1001 \\
{[\mathrm{M}+\mathrm{HOAC}-} \\
\mathrm{H}] \rightarrow 941\end{array}$ & $\begin{array}{l}\text { Full MS } \\
\text { 1001, 979, 941, 885, } \\
798,601,547,477,283 \text {, } \\
179\end{array}$ & Asiaticoside D & CA & - & - & - \\
\hline 34 & 38.48 & - & $\begin{array}{l}1307 \\
{[\mathrm{M}+\mathrm{HOAc}-} \\
\mathrm{H}] \rightarrow 1248\end{array}$ & $\begin{array}{l}\mathrm{MS}^{2} \mathbf{1 2 4 8}, 1191,1029 \\
854,84 \\
\mathrm{MS}^{3} \mathbf{1 0 8 7}, 729\end{array}$ & $\begin{array}{l}\text { Ranuncoside IV with addition of } \\
\text { glucose unit }\end{array}$ & $\mathrm{HB}$ & - & - & - \\
\hline 35 & 38.97 & - & $\begin{array}{l}1145.3 \\
{[\mathrm{M}+\mathrm{HOAc}-} \\
\mathrm{H}] \rightarrow 1085\end{array}$ & $\begin{array}{l}\text { Full MS } \\
\mathbf{1 1 4 5}, 1085,996,923, \\
853,787,731,643,588, \\
529,506,495,483,357 \\
275,179,173,132,119\end{array}$ & Ranuncoside IV & $\mathrm{HB}$ & - & - & - \\
\hline 36 & 39.51 & - & $\begin{array}{l}1145 \\
{[\mathrm{M}+\mathrm{HOAc}-} \\
\mathrm{H}] \rightarrow 1085\end{array}$ & $\begin{array}{l}\text { Full MS } \\
\mathbf{1 1 4 5}, 1086,985,965, \\
952,855,810,793,601, \\
573,506,468,289,193, \\
178,165,151,147,119\end{array}$ & Ranuncoside V & $\mathrm{HB}$ & - & - & - \\
\hline 37 & 40.03 & - & $\begin{array}{l}563 \\
{[\mathrm{M}+\mathrm{HOAC}-} \\
\mathrm{H}] \rightarrow 503\end{array}$ & $\begin{array}{l}\mathrm{MS}^{2} \mathbf{5 0 5}, 283 \\
\mathrm{MS}^{3} \mathbf{4 3 5}, 426,390\end{array}$ & Madecassic acid & $\mathrm{CA}$ & - & - & - \\
\hline 38 & 41.27 & - & 956 & $\begin{array}{l}\mathrm{MS}^{2} 835, \mathbf{7 3 2}, 714,617 \\
569,549,524,456,319\end{array}$ & Hydrocotyloside II & HS & - & - & - \\
\hline 39 & 41.71 & - & $\begin{array}{l}547.2 \\
{[\mathrm{M}+\mathrm{HOAC}-} \\
\mathrm{H}] \rightarrow 487\end{array}$ & $\begin{array}{l}\mathrm{MS}^{2} \mathbf{4 8 7}, 466,427,362 \\
\mathrm{MS}^{3} 454,440, \mathbf{4 0 8}\end{array}$ & Asiatic acid & $\mathrm{CA}$ & & & \\
\hline \multicolumn{10}{|l|}{ Unknown } \\
\hline 9 & 13.09 & 250,310 & 693 & $\begin{array}{l}\mathbf{6 7 5}, 643,337, \mathbf{5 9 9}, 547 \\
369\end{array}$ & & $\mathrm{HB}$ & - & - & - \\
\hline 27 & 32.16 & - & 919 & 799, 720, 645, 263 & & HS & - & - & - \\
\hline 40 & 43.72 & 240,285 & $\begin{array}{l}735 \\
{[\mathrm{M}+\text { HOAC- }} \\
\mathrm{H}] \rightarrow 676\end{array}$ & $675, \mathbf{6 5 9}, 586,440$ & & HB & - & - & - \\
\hline 41 & 45.35 & $260,340,405$ & $\begin{array}{l}737 \\
{[\mathrm{M}+\mathrm{HOAC}-} \\
\mathrm{H}] \rightarrow 677\end{array}$ & $719, \mathbf{6 7 4}, 524,473,296$ & & $\mathrm{HB}$ & - & - & - \\
\hline 42 & 45.46 & 255,425 & 293 & $\mathbf{2 7 5}, 236,171,163$ & & HS & - & - & - \\
\hline 43 & 47.51 & 250,335 & 295 & $295,277,185, \mathbf{1 7 1}, 151$ & & $\mathrm{HB}, \mathrm{HS}$ & - & - & - \\
\hline
\end{tabular}

a C. asiatica (CA); H. bonariensis (HB); H. sibthorpioides (HS).

b Value are means $(n=3) \pm \%$ RSD.

c The amount of compound was relative to amount of standard chlorogenic acid, with relationship between peak areas $(y)$ and concentrations $(x)$ was $y=269319.45 x+1566573.47\left(R^{2}=0.990\right)$.

d The amount of compound was relative to amount of standard quercetin, with relationship between peak areas $(y)$ and concentrations $(x)$ was $y=237399 x-877718\left(R^{2}=0.999\right)$. 


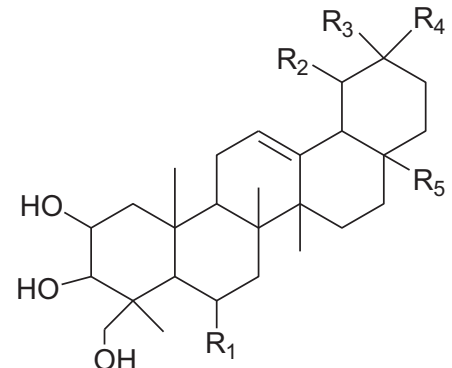

21

23

26

28

37

39

$$
\begin{aligned}
& \mathrm{R}_{1}=\mathrm{OH} ; \mathrm{R}_{2}=\mathrm{CH}_{3} ; \mathrm{R}_{3}=\mathrm{CH}_{3} ; \mathrm{R}_{4}=\mathrm{H} ; \mathrm{R}_{5}=\mathrm{COO}-\operatorname{glc}(1-6) \operatorname{glc}(1-4) \mathrm{rha} \\
& \mathrm{R}_{1}=\mathrm{OH} ; \mathrm{R}_{2}=\mathrm{H} ; \mathrm{R}_{3}=\mathrm{CH}_{3} ; \mathrm{R}_{4}=\mathrm{CH}_{3} ; \mathrm{R}_{5}=\mathrm{COO}-\operatorname{glc}(1-6) \operatorname{glc}(1-4) \mathrm{rha} \\
& \mathrm{R}_{1}=\mathrm{H} ; \mathrm{R}_{2}=\mathrm{H} ; \mathrm{R}_{3}=\mathrm{CH}_{3} ; \mathrm{R}_{4}=\mathrm{CH}_{3} ; \mathrm{R}_{5}=\mathrm{COO} \text {-glc(1-6)glc(1-4)rha } \\
& \mathrm{R}_{1}=\mathrm{H} ; \mathrm{R}_{2}=\mathrm{CH}_{3} ; \mathrm{R}_{3}=\mathrm{CH}_{3} ; \mathrm{R}_{4}=\mathrm{H} ; \mathrm{R}_{5}=\mathrm{COO} \text {-glc(1-6)glc(1-4)rha } \\
& \mathrm{R}_{1}=\mathrm{OH} ; \mathrm{R}_{2}=\mathrm{CH}_{3} ; \mathrm{R}_{3}=\mathrm{CH}_{3} ; \mathrm{R}_{4}=\mathrm{H} ; \mathrm{R}_{5}=\mathrm{H} \\
& \mathrm{R}_{1}=\mathrm{H} ; \mathrm{R}_{2}=\mathrm{CH}_{3} ; \mathrm{R}_{3}=\mathrm{CH}_{3} ; \mathrm{R}_{4}=\mathrm{H} ; \mathrm{R}_{5}=\mathrm{H}
\end{aligned}
$$

Fig. 3. Compounds identified from C. asiatica.

the deprotonated molecular ion peak at $m / z 625$ and the fragment ions at $\mathrm{m} / \mathrm{z} 463$ and 301 attributable to two consecutive losses of a glucose moiety. Kaempferol-3-O-rhamnoside was also detected in $H$. sibthorpioides extract (peak 19, $\mathrm{RT}=19.17 \mathrm{~min}$ ) with deprotonated molecular ion at $m / z 431$ and fragment ions at $m / z 285$ characteristic of the aglycone, kaempferol.

\subsection{Identification of triterpene glycosides}

Pentacyclic triterpenes have been reported to occur in Centella species either as oleanane or ursane types, with the latter being the more common of the two types. Similarly in our LCMS/MS analysis, ursane triterpenes were detected in C. asiatica extract. The TIC profile of $C$. asiatica extract showed four major peaks attributable to madecassoside (peak 21), asiaticoside B (peak 23), centellasaponin A (peak 26), asiaticoside (peak 28), asiaticoside D (peak 32) and madecassic acid (peak 37). The structures for these compounds are illustrated in Fig. 3. Identification of the triterpene glycosides in $C$. asiatica extract were supported by comparison with the full MS of authentic standards (madecassoside, asiaticoside, madecassic acid and asiatic acid) as well as by comparison with literature data (Long et al., 2012; Shen et al., 2009). Their full MS spectrum (negative ion mode) showed the pseudo-molecular ions $\left([\mathrm{M}+\mathrm{HOAc}-\mathrm{H}]^{-}\right.$of each triterpene glycosides, but MS/MS experiments failed to produce any fragment ions. Peak $\mathbf{2 6}$ was tentatively identified as the oleanane type triterpene, Centellasaponin A, an isomer of asiaticoside, mainly based on its fragmentation pattern. Additionally, $C$. asiatica exhibited a minor constituent (peak 39) which exhibited a pseudo-molecular ion peak at $m / z 547$ for $[\mathrm{M}+\mathrm{HOAc}-\mathrm{H}]^{-}$, assigned as asiatic acid based on its fragmentation pattern and comparison with authentic standard.

In contrast to $C$. asiatica, the triterpene glycosides reported from the Hydrocotyle extracts were mostly of the oleanane-type. These findings were in accordance with previous literature (Greca et al., 1994; Matsushita et al., 2004). The TIC profile for $H$. bonariensis extract displayed a pseudo-molecular ion at $m / z 1021>[\mathrm{M}+\mathrm{HOAc}-$ $\mathrm{H}]^{-}$for peak $16(\mathrm{RT}=18.12 \mathrm{~min})$ and $\mathrm{MS} / \mathrm{MS}$ fragments ions at $\mathrm{m} / \mathrm{z} 799$ for $[\mathrm{M}-\mathrm{H}-162]^{-}, 667$ for [M-H-162-132] $^{-}$and 505 for $[\mathrm{M}-\mathrm{H}-(2 \times 162)-132]^{-}$, indicating the sequential losses of glucose, arabinose and glucose moieties, respectively. The fragment at $\mathrm{m} / \mathrm{z}$ 505 matched that of the aglycone $\mathrm{R}_{1}$-barrigenol. Comparison with literature data (Greca et al., 1994) supported the assignment of this component as ranuncoside II.

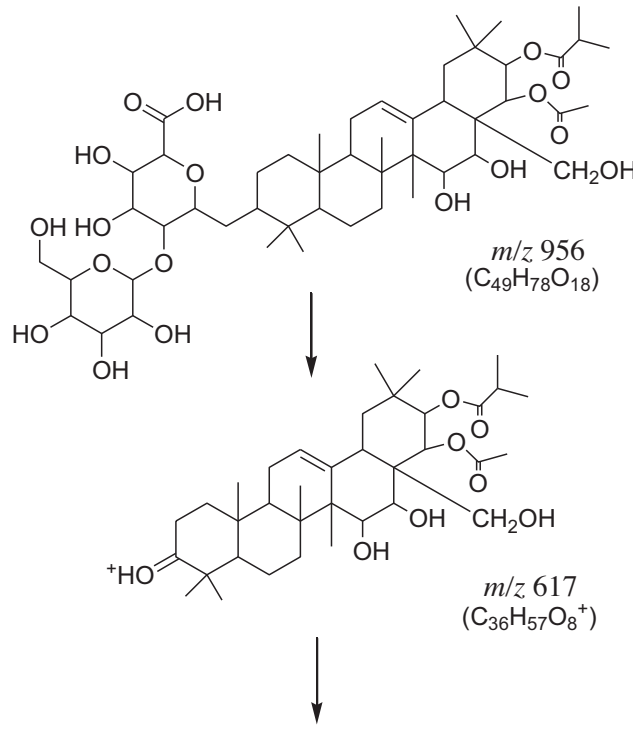<smiles>C=COC1C(OC=C(C)C)C(C)(C)CC2C3=CCC4C5(C)CCCC(C)(C)C5CCC4(C3C)C(O)C(O)C21CO</smiles>

Fig. 4. Proposed mass fragmentation pathway for Hydroctyloside II.

Ranuncoside 1 appeared as peak $20(\mathrm{RT}=21.15 \mathrm{~min})$ which yielded a pseudo-molecular ion peak at $m / z 859\left([\mathrm{M}+\mathrm{HOAc}-\mathrm{H}]^{-}\right)$ and fragment ion peak at $m / z 505$ in the MS/MS spectra corresponded to its aglycone, resulting from losses of a pentose and a glucose units. The pseudo-molecular ion at $m / z 1307$ ([M+HOAc$\mathrm{H}]^{-}$) for peak $34(\mathrm{RT}=38.48 \mathrm{~min})$, indicated a structure consistent with ranuncoside IV (MW 1086) with an additional glucose unit (MW 162). Peak 35 (RT=38.97 $\mathrm{min}$ ) and $36(\mathrm{RT}=39.51 \mathrm{~min})$ were isomeric, showing similar pseudo-molecular ions at $\mathrm{m} / \mathrm{z} 1145$ $[\mathrm{M}+\mathrm{HOAc}-\mathrm{H}]^{-}$. They were tentatively assigned as ranuncoside IV and ranuncoside V (Greca et al., 1994), respectively, based on their full MS data. The presence of other ranuncosides was also detected in $H$. bonariensis. Several other triterpene glycosides with $\mathrm{R}_{1}$-barrigenol as its aglycone were also detected in $\mathrm{H}$. bonariensis. These are peaks $22(\mathrm{RT}=24.19 \mathrm{~min})$ and $\mathbf{2 5}(\mathrm{RT}=28.24 \mathrm{~min})$, which gave pseudo-molecular ions at $\mathrm{m} / z 946[\mathrm{M}+\mathrm{HOAc}-\mathrm{H}]^{-}$and 813 $[\mathrm{M}+\mathrm{HOAc}-\mathrm{H}]^{-}$, respectively.

Oleanane glycosides were also detected in $H$. sibthorpioides extract with barrigenol as its aglycone, but with propanoyl or methylpropanoyl and acetyl groups attached at C21 and C22, respectively (Matsushita et al., 2004). Peak 38 (RT = $41.27 \mathrm{~min}$ ) gave a deprotonated molecular ion peak at $\mathrm{m} / \mathrm{z} 956$ and fragment ion at $\mathrm{m} / \mathrm{z} 617$ corresponding to the losses of a glucose and a hexosyluronic acid [M-H-162-176] $]^{-}$. Furthermore, the MS/MS showed a fragment ion at $m / z 569$ indicating the barrigenol aglycone minus a methoxy group and water $\left[\mathrm{M}-\mathrm{H}-\mathrm{OCH}_{3}-\mathrm{H}_{2} \mathrm{O}\right]^{-}$. Thus, the compound was assigned as hydrocotyloside II (Fig. 4). Peak 31 (RT = $37.27 \mathrm{~min}$ ) which gave a deprotonated molecular ion at $\mathrm{m} / \mathrm{z} 1118$ and a fragment ion at $m / z 956$ for $[\mathrm{M}-\mathrm{H}-162]^{-}$was assigned as hydrocotyloside III. The retention times, UV, MS and $\mathrm{MS}^{2}$ data obtained by HPLC-DAD-ESIMS, as well as the relative amounts of antioxidant compounds of each of the Pegaga varieties are presented in Table 1. 
Table 2

Total phenolic content (TPC), radical scavenging activity (RSA), and xanthine oxidase inhibitory (XOI) activities of three Pegaga extracts.

\begin{tabular}{llll}
\hline Sample & $\begin{array}{l}\text { TPC } \\
(\mathrm{mg} \mathrm{GAE} / 100 \mathrm{~g}\end{array}$ & $\begin{array}{l}\text { DPPH-RSA } \\
(\text { at } \\
\text { DW })^{\mathrm{a}, \mathrm{b}}\end{array}$ & $\begin{array}{l}\text { XOI } \\
(\text { at }\end{array}$ \\
& $72.09 \pm 2.45$ & $72.99 \pm 0.42$ & $87.68 \pm 4.08$ \\
\hline C. asiatica & $28.55 \pm 1.14$ & $22.43 \pm 0.90$ & $32.23 \pm 1.28$ \\
H. bonariensis & $56.23 \pm 2.33$ & $37.86 \pm 0.49$ & $14.73 \pm 1.06$ \\
H. sibthorpioides & & & $84.68 \pm 1.25$ \\
Allopurinol $(10 \mu \mathrm{M})$ & & $87.63 \pm 1.63$ & \\
Quercetin $(50 \mu \mathrm{M})$ & & \\
\hline
\end{tabular}

a The experiment was conducted in triplicates and the result was calculated as mean \pm SEM.

b For TPC assay, the curve absorbance of standard gallic acid versus concentrations is described by the equation $y=0.0059 x+0.0291\left(R^{2}=0.996\right)$.

\subsection{Determination of the total phenolic content and antioxidant activity}

Phenolic compounds are often correlated to the antioxidant properties of a plant due to their capability as electron donors in free radical reactions. In this study, using the Folin-Ciocalteu method, it was shown that there are significant differences $(p<0.05)$ in the TPCs of the Pegaga extracts. The TPCs of the extracts ranged from 28.6 to $77.9 \mathrm{mg}$ GAE/100 $\mathrm{g}$ DW with $C$. asiatica showing the highest value of $77.9 \mathrm{mg}$ GAE/100 $\mathrm{g}$ DW, followed by $H$. sibthorpioides and $H$. bonariensis with TPC values of 58.2 and $28.6 \mathrm{mg} \mathrm{GAE} / 100 \mathrm{~g}$ DW, respectively (Table 2 ).

Reactive oxygen species (ROS) play an important role in ageing and age-related diseases. Thus, taking antioxidants as supplements are expected to slow down the ageing process. In this study, determination of the antioxidant activities of the three Pegaga extracts $(C$. asiatica, $H$. bonariensis, and $H$. sibthorpioides) were carried out based on DPPH radical scavenging activity (RSA) and xanthine oxidase inhibitory assays. The mechanism involved in DPPH assay is that the stable free radical (DPPH) will react with antioxidants to form 1,1diphenyl-2-picrylhydrazine. The presence of free radical-scavenger results in the disappearance of color and therefore its high radical scavenging percentage represents high antioxidant activity. Adopting this experiment to our samples, $C$. asiatica extract was found to be the most potent radical scavenger towards DPPH with $\mathrm{IC}_{50}$ value of $180.3 \mu \mathrm{g} / \mathrm{ml}$, while extracts $H$. bonariensis and $H$. sibthorpioides showed weaker activity with $\mathrm{IC}_{50}$ value of $>250.0 \mu \mathrm{g} / \mathrm{ml}$. Further ANOVA test with Tukey-HSD pairwise comparison showed that there is significant difference $(p<0.05)$ in DPPH-RSA of $C$. asiatica when compared to $H$. bonariensis and $H$. sibthorpioides extracts (Table 2). The antioxidant results were in good correlation with the TPC values. These results were also supported by quantitative determination of antioxidant compounds using HPLC-DAD quantitative analyses. $C$. asiatica extract has the highest amounts of phenolics $(0.119 \mathrm{mg} / 100 \mathrm{~g}$ of extract in the forms of caffeoylquinic acid derivatives) and flavonoids ( $1.088 \mathrm{mg} / 100 \mathrm{~g}$ of extract), followed by $H$. sibthorpioides with total phenolics of $0.018 \mathrm{mg} / 100 \mathrm{~g}$ of extract and total flavonoids of $0.807 \mathrm{mg} / 100 \mathrm{~g}$ of extract, and $H$. bonariensis extracts with total flavonoids of $0.759 \mathrm{mg} / 100 \mathrm{~g}$ of extract (Table 1). According to our previous study on the application of Partial Least Squares model for the prediction of antioxidant activity in Pegaga extracts from ${ }^{1} \mathrm{H}$ NMR spectroscopy, dicaffeoylquinic acid derivatives were the constituents that gave the most contribution to the DPPH-RSA, followed by quercetin and kaempferol derivatives (Maulidiani et al., 2013).

Xanthine oxidase (XO) is a key enzyme that catalyzes the last two steps of purine catabolism, the oxidation of hypoxanthine to xanthine and xanthine to uric acid. Elevation of uric acid level in the blood will leads to the deposition of urate crystals in joints and kidneys causing hyperuricemia and gout arthritis. The results from the assay showed that $C$. asiatica extract inhibited $87.7 \%$ XO activity at the concentration of extract $200 \mu \mathrm{g} / \mathrm{ml}$, while the two species of Hydrocotyle showed weak inhibitory activity (Table 2). Furthermore, ANOVA test with Tukey-HSD pairwise comparison showed that there is significant $(p<0.05)$ difference of XO inhibitory activity between C. asiatica and the other two Hydrocotyle extracts (Table 2).

\section{Conclusion}

In conclusion, identification of the three Pegaga (C. asiatica, $H$. bonariensis, and $H$. sibthorpioides) varieties using LC-DAD-ESIMS/MS technique allowed us to characterize the chemical constituents of the plants based on their MS data and MS/MS fragmentation pattern. In addition to quantification of the total phenolic content, the identity of the chlorogenic acids, flavonoids, and triterpenes composition in the three varieties was also determined. Antioxidant evaluation (DPPH-RSA, and XOI activities) of the extracts showed that the potency correlated well with the total phenolic content (TPC) and revealed that $C$. asiatica extract was the most active compared to $H$. bonariensis, and $H$. sibthorpioides. Based on this observation, with respect to the medicinal use of Pegaga as an antioxidant agent, $C$. asiatica would be the more preferred species. In addition, LC-DAD-ESI/MS analysis of the Pegaga extracts, might be one of the alternative solution for quality control and standardization of the plants.

\section{Acknowledgements}

The authors thank Universiti Putra Malaysia for financial support this project under Research University Grant Scheme (project no. 05/01/07/0178RU). NHL also thanks the Scientific Chairs Unit, Taibah University (KSA) for its supports.

\section{References}

Al-Saeedi, F.J., Bitar, M., Pariyani, S., 2011. Effect of asiaticoside on 99mTctetrofosmin and 99mTc-sestamibi uptake in MCF-7 cells. J. Nucl. Med. Technol. 39, 279-283

Asakawa, Y., Matsuda, R., Takemoto, T., 1982. Mono- and sesquiterpenoids from Hydrocotyle and Centella species. Phytochemistry 21, 2590-2592.

Brinkhaus, B., Lindner, M., Schuppan, D., Hahn, E.G., 2000. Chemical, pharmacological and clinical profile of the East Asian medical plant Centella asiatica. Phytomedicine 7, 427-448

Burkill, I.H., 1966. Dictionary of the Economic Products of the Malay Peninsula. Ministry of Agriculture and Cooperatives, Kuala Lumpur, Malaysia.

De Padua, L.S., Bunyapraphatsara, N., 1999. Medicinal and Poisonous Plants 1. In Plant Resources of South-East Asia. Backhuys, Leiden, The Netherlands.

Ferreres, F., Taveira, M., Pereira, D.M., Valentao, P., Andrade, P.B., 2010. Tomato (Lycopersicon esculentum) seeds: new flavonols and cytotoxic effect. J. Agric. Food Chem. 58, 2854-2861.

Funk, C., Ralph, J., Steinhart, H., Bunzel, M., 2005. Isolation and structural characterisation of 8-0-4/8-0-4- and 8-8/8-0-4-coupled dehydrotriferulic acids from maize bran. Phytochemistry 66, 363-371.

Govindan, G., Sambandan, T.G., Govindan, M., Sinskey, A., Vanessendelft, J., Adenan, I., Rha, C.K., 2007. A bioactive polyacetylene compound isolated from Centella asiatica. Planta Med. 73, 597-599.

Greca, M.D., Fiorentino, A., Monaco, P., Previtera, L., 1994. Oleanane glycosides from Hydrocotyle ranunculoides. Phytochemistry 36, 1479-1483.

Gunther, B., Wagner, H., 1996. Quantitative determination of triterpenes in extracts and phytopreparations of Centella asiatica (L.) Urban. Phytomedicine 3, 59-65.

Kim, H.K., Choi, Y.H., Verpoorte, R., 2010. NMR-based metabolomic analysis of plants. Nat. Protoc. 5, 536-549.

Lin, L.-Z., Harnly, J.M., 2010. Identification of the phenolic components of chrysanthemum flower (Chrysanthemum morifolium Ramat). Food Chem. 120, 319-326.

Lindon, J.C., Nicholson, J.K., 1997. Recent advances in high-resolution NMR spectroscopic methods in bioanalytical chemistry. TrAC, Trends Anal. Chem. 16, 190-200.

Long, H.S., Stander, M.A., Van Wyk, B.E., 2012. Notes on the occurrence and significance of triterpenoids (asiaticoside and related compounds) and caffeoylquinic acids in Centella species. S. Afr. J. Bot. 82, 53-59.

Matsuda, H., Morikawa, T., Ueda, H., Yoshikawa, M., 2001. Medicinal foodstuffs. XXVII. Saponin constituents of gotu kola (2): structures of new ursane- and oleanane-type triterpene oligoglycosides, centellasaponins B, C, and D, from Centella asiatica cultivated in Sri Lanka. Chem. Pharm. Bull. 49, 1368-1371. 
Matsushita, A., Sasaki, Y., Warashina, T., Miyase, T., Noguchi, H., Velde, D.V., 2004. Hydrocotylosides I-VII, New oleanane saponins from Hydrocotyle sibthorpioides. J. Nat. Prod. 67, 384-388.

Maulidiani, H., Khatib, A., Shaari, K., Abas, F., Shitan, M., Kneer, R., Neto, V., Lajis, N.H., 2012. Discrimination of three pegaga (Centella) varieties and determination of growth-lighting effects on metabolites content based on the chemometry of ${ }^{1} \mathrm{H}$ nuclear magnetic resonance spectroscopy. J. Agric. Food Chem. 60, 410-417.

Maulidiani, Abas, F., Khatib, A., Shitan, M., Shaari, K., Lajis, N.H., 2013. Comparison of partial least squares and artificial neural network for the prediction of antioxidant activity in extract of Pegaga (Centella) varieties from ${ }^{1} \mathrm{H}$ nuclear magnetic resonance spectroscopy. Food Res. Int. 54, 852-860.

Mohamad, H., Abas, F., Permana, D., Lajis, N.H., Ali, A.M., Sukari, M.A., Hin, T.Y.Y., Kikuzaki, H., Nakatani, N., 2004. DPPH free radical scavenger components from the fruits of Alpinia rafflesiana Wall. ex Bak. (Zingiberaceae). Z. Naturforsch. C J. Biosci. 59, 811-815.

Oblong, J.E., Bissett, D.L., 1997. Methods of regulating skin condition with Centella asiatica extract. In: Patent Cooperation Treaty. W. I. P. Organization, pp. 1-45.

Rafamantanana, M.H., Rozet, E., Raoelison, G.E., Cheuk, K., Ratsimamanga, S.U., Hubert, P., Quetin-Leclercq, J., 2009. An improved HPLC-UV method for the simultaneous quantification of triterpenic glycosides and aglycones in leaves of Centella asiatica (L.) Urb (APIACEAE). J. Chromatogr. B: Anal. Technol. Biomed. Life Sci. 877, 2396-2402.

Rastogi, R.P., Sakar, B., Dhar, M.L., 1960. Chemical examination of Centella asiatica Linn Part I. Isolation of the chemical constituents. J. Sci. Ind. Res. 19, 252-257.

Satake, T., Kamiya, K., An, Y., Oishi Nee Taka, T., Yamamoto, J., 2007. The antithrombotic active constituents from Centella asiatica. Biol. Pharm. Bull. 30, 935-940.
Schaneberg, B.T., Mikell, J.R., Bedir, E., Khan, I.A., 2003. An improved HPLC method for quantitative determination of six triterpenes in Centella asiatica extracts and commercial products. Pharmazie 58, 381-384.

Schutz, K., Kammerer, D., Carle, R., Schieber, A., 2004. Identification and quantification of caffeoylquinic acids and flavonoids from artichoke (Cynara scolymus L.) heads, juice, and pomace by HPLC-DAD-ESI/MSn. J. Agric. Food Chem. 52, 4090-4096.

Seeram, N.P., Lee, R., Scheuller, H.S., Heber, D., 2006. Identification of phenolic compounds in strawberries by liquid chromatography electrospray ionization mass spectroscopy. Food Chem. 97, 1-11.

Sene, G., Loiseau, A., Lepetit, J., 2007. Use of compounds from Centella asiatica. In: Patent Cooperation Treaty. W. P. I. Organization, pp. 1-18.

Shen, Y., Liu, A., Ye, M., Wang, L., Chen, J., Wang, X.R., Han, C., 2009. Analysis of biologically active constituents in Centella asiatica by microwave-assisted extraction combined with LC-MS. Chromatographia 70, 431-438.

Singh, A.P., Wilson, T., Luthria, D., Freeman, M.R., Scott, R.M., Bilenker, D., Shah, S., Somasundaram, S., Vorsa, N., 2011. LC-MS-MS characterisation of curry leaf flavonols and antioxidant activity. Food Chem. 127, 80-85.

Singleton, V.L., Orthofer, R., Lamuela-Raventos, R.M., Lester, P., 1999. Analysis of total phenols and other oxidation substrates and antioxidants by means of folinciocalteu reagent. Methods Enzymol. 299, 152-178.

Sweeney, A.P., Wyllie, S.G., Shalliker, R.A., Markham, J.L., 2001. Xanthine oxidase inhibitory activity of selected Australian native plants. J. Ethnopharmacol. 75, 273-277.

Wan, J., Gong, X., Jiang, R., Zhang, Z., Zhang, L., 2012. Antipyretic and antiinflammatory effects of asiaticoside in lipopolysaccharide-treated rat through up-regulation of heme oxygenase-1. Phytother. Res. 27, 1136-1142. 